\title{
Targeting rehabilitation to improve outcomes after total knee arthroplasty in patients at risk of poor outcomes: randomised controlled trial
}

\author{
David F Hamilton, ${ }^{1}$ David J Beard, ${ }^{2}$ Karen L Barker, ${ }^{2}$ Gary J Macfarlane, ${ }^{3}$ Christopher E Tuck, ${ }^{4}$ \\ Andrew Stoddart, ${ }^{4}$ Timothy Wilton, ${ }^{5}$ James D Hutchinson, ${ }^{6}$ Gordon D Murray, ${ }^{4}$ \\ A Hamish R W Simpson, ${ }^{1}$ on behalf of the TRIO investigators
}

For numbered affiliations see end of the article.

Correspondence to: D F Hamilton d.f.hamilton@ed.ac.uk (or@df hamilton on Twitter: ORCID 0000-0001-9060-9255) Additional material is published online only. To view please visit the journal online.

Cite this as: BMJ 2020;371:m3576 http://dx.doi.org/10.1136/bmj.m3576 Accepted: 24 August 2020

\section{ABSTRACT}

OBJECTIVE

To evaluate whether a progressive course of outpatient physiotherapy offers superior outcomes to a single physiotherapy review and home exercise based intervention when targeted at patients with a predicted poor outcome after total knee arthroplasty. DESIGN

Parallel group randomised controlled trial.

SETTING

13 secondary and tertiary care centres in the UK providing postoperative physiotherapy.

PARTICIPANTS

334 participants with knee osteoarthritis who were defined as at risk of a poor outcome after total knee arthroplasty, based on the Oxford knee score, at six weeks postoperatively. 163 were allocated to therapist led outpatient rehabilitation and 171 to a home exercise based protocol.

\section{INTERVENTIONS}

All participants were reviewed by a physiotherapist and commenced 18 sessions of rehabilitation over six weeks, either as therapist led outpatient rehabilitation (progressive goal oriented functional rehabilitation protocol, modified weekly in one-one contact sessions) or as physiotherapy review followed by a home exercise based regimen (without progressive input from a physiotherapist).

\section{WHAT IS ALREADY KNOWN ON THIS TOPIC}

Despite physiotherapy after total knee arthroplasty being considered important for optimal results, rehabilitation varies worldwide

Differing postoperative rehabilitation approaches have not been shown to deliver meaningful differences in patient outcomes after surgery

This finding is based on physiotherapy applied to all patients, so it is unclear whether people at risk of poor recovery would benefit from more intensive rehabilitation

\section{WHAT THIS STUDY ADDS}

This study suggests that targeting postoperative physiotherapy to those most in need is a feasible delivery method, but that the actual content of the rehabilitation seems to have minimal influence on patient outcomes

That there was no additional benefit of physical outpatient rehabilitation compared with single physiotherapist review and home exercise based could have implications for healthcare delivery and resource planning around this high volume procedure

\section{MAIN OUTCOME MEASURES}

Primary outcome was Oxford knee score at 52 weeks, with a 4 point difference between groups considered to be clinically meaningful. Secondary outcomes included additional patient reported outcome measures of pain and function at 14, 26, and 52 weeks post-surgery.

\section{RESULTS}

334 patients were randomised. Eight were lost to follow-up. Intervention compliance was more than $85 \%$. The between group difference in Oxford knee score at 52 weeks was 1.91 (95\% confidence interval -0.18 to 3.99) points, favouring the outpatient rehabilitation $\operatorname{arm}(\mathrm{P}=0.07)$. When all time point data were analysed, the between group difference in Oxford knee score was a non-clinically meaningful 2.25 points ( 0.61 to $3.90, P=0.01$ ). No between group differences were found for secondary outcomes of average pain ( 0.25 points, -0.78 to $0.28, \mathrm{P}=0.36$ ) or worst pain ( 0.22 points, -0.71 to $0.41, \mathrm{P}=0.50$ ) at 52 weeks or earlier time points, or of satisfaction with outcome (odds ratio 1.07, 95\% confidence interval 0.71 to $1.62, \mathrm{P}=0.75$ ) or post-intervention function (4.64 seconds, $95 \%$ confidence interval -14.25 to 4.96, $\mathrm{P}=0.34)$.

\section{CONCLUSIONS}

Outpatient therapist led rehabilitation was not superior to a single physiotherapist review and home exercise based regimen in patients at risk of poor outcomes after total knee arthroplasty. No clinically relevant differences were observed across primary or secondary outcome measures.

\section{TRIALS REGISTRATION}

Current Controlled Trials ISRCTN23357609 and ClinicalTrials.gov NCT01849445.

\section{Introduction}

Total knee arthroplasty is a common procedure for end stage osteoarthritis of the knee, with more than 100000 knee arthroplasties performed each year in the United Kingdom ${ }^{1}$ and more than 700000 in the United States. ${ }^{2}$ Projections of future surgical volumes suggest further substantial increases..$^{3-5}$ Although total knee arthroscopy is effective at reducing pain and improving physical function for most patients, around $20 \%$ report dissatisfaction with the postoperative outcome. $^{26}$

Physiotherapy is generally thought to be important in achieving optimal results after knee arthroplasty, yet the content of rehabilitation varies worldwide. ${ }^{7}$ Some 
countries such as the US and Australia use prolonged postoperative inpatient rehabilitation, although the effectiveness of this in improving outcomes (compared with outpatient based physiotherapy) has been questioned. ${ }^{8}$ In the UK, inpatient rehabilitation is of short duration, with patients typically discharged 3-5 days after arthroplasty. Under this model, physiotherapy during the brief inpatient stay is aimed at encouraging mobilisation and facilitating a safe discharge. Subsequent rehabilitation provision after discharge varies widely ${ }^{910}$ and no definitive guidelines exist for rehabilitation after knee replacement. The generally held assumption that increased therapist contact enhances the rehabilitation has recently been challenged. Meta-analyses suggest that uniform postoperative physiotherapy for all patients after total knee arthroplasty compared with no treatment offers short term benefit but is not effective at improving patient outcomes at one year. ${ }^{11-13}$ Further recent metaanalysis of randomised trials suggests no difference in patient outcomes when rehabilitation consists of home or outpatient based interventions after total knee arthroplasty (with conclusions based on weak to moderate evidence). ${ }^{14} 15$ However, as most patients report a good result after total knee arthroplasty, it might be that subgroups of patients could benefit from a targeted physiotherapy intervention. We determined if a six week programme of outpatient physiotherapy offers superior outcomes to a home exercise based regimen when targeted, in the early postoperative phase, at patients with predicted poor recovery and clinical outcomes after total knee arthroplasty.

\section{Methods}

The TRIO (Targeted Rehabilitation to Improve Outcome) study was a multicentre parallel group randomised controlled trial evaluating the effect of different physiotherapy interventions targeted at patients with poor recovery six weeks after total knee arthroplasty. The study was carried out according to the published protocol. $^{16}$

The study was registered before participant recruitment, which began in September 2013. The registration documents lacked detail of the planned secondary outcomes, which were detailed in the trial protocol document (published in the first months of recruitment). Because it is important to report as per the trial registration, both the secondary outcomes and the planned secondary outcomes that were not reported in the trial registration documents are provided separately in the results. As we planned to collect all data at the onset of recruitment, data on baseline outcomes are available for all trial participants.

\section{Recruitment and consent}

Participants were recruited from 13 hospitals that provide total knee arthroplasty in the UK. Patients underwent routine surgical procedures at the study centres, which utilise local standard implants and techniques. All patients received immediate postoperative physiotherapy on the hospital wards, promoting mobility and knee range of motion and aimed at safe hospital discharge. Patients were screened for trial eligibility at routine clinical review six weeks after total knee arthroplasty. To be eligible for the study, patients had to have undergone a primary total knee arthroplasty for osteoarthritis and be at risk of a poor outcome (defined as an Oxford knee score of $\leq 26$ points, ${ }^{17}{ }^{18}$ completed at that six week postoperative time point). The Oxford knee score measures pain and function after knee arthroplasty. The defined cut point of 26 points ${ }^{17}$ highlights those patients with most knee specific pain and dysfunction from simple activities, such as raising from a chair or mobilising a short distance. We excluded patients who were unwilling or unable to comply with the rehabilitation protocols, underwent arthroplasty purely for pain relief (ie, those with no expectation of mobilising postoperatively), required complex revision procedures, could not, or were unwilling to, attend their local outpatient department for rehabilitation, or had already received structured ongoing outpatient physiotherapy at six weeks post-surgery. Research nurses screened patients at the local sites and completed screening logs to record reasons for ineligibility.

\section{Randomisation and protection against bias}

The local team carried out randomisation through a secure web based service supported by the Edinburgh Clinical Trials Unit. Randomisation was on a 1:1 basis, stratified by centre, with block allocation. As the intervention groups had clearly differing types and locations of physiotherapy, participants could not be masked to group assignment. The physiotherapists delivering the intervention could not be blinded to the intervention and thus were not involved in the study assessments beyond recording the timed-get-up-andgo test before and after the treatment intervention. Participants self-reported the primary outcome and secondary outcomes. To minimise bias, statistical analysis was carried out blinded to allocation.

\section{Interventions}

All participants were reviewed by a physiotherapist two weeks after recruitment (eight weeks after total knee arthroplasty), at which point they were provided with rehabilitation advice and education about recovery, pain management, and pacing of activities. The participants then commenced 18 exercise sessions over six weeks and documented their exercise in a rehabilitation diary. On completion of the six week intervention all participants received a final session with the physiotherapist to review progress. The exercise diary was reviewed at the end of this study intervention visit and adherence was documented. After completion of the trial interventions at week 14, participants were advised to continue to progress their rehabilitation and exercise levels independently over the postoperative year. The published protocol includes full details of the trial interventions. $^{16}$ 


\section{Outpatient therapist led rehabilitation}

The outpatient therapist led group undertook a progressive functional rehabilitation protocol (reviewed and modified weekly in a one-to-one contact session). The specific rehabilitation intervention for this study was based on best evidence for functional outcome. ${ }^{19}$ We used a goal led protocol that incorporated four categories: range of motion, strengthening, proprioception, and walking gait. Rehabilitation focused on agreed patient goals within these categories, with amendments over the six weeks as required. Although target goals were suggested for each category, the physiotherapists had flexibility in deciding how the goals could be achieved using local facilities and equipment, with a focus on exercise rehabilitation. Participants undertook a further two sessions of personalised rehabilitation each week at home. The physiotherapist directed this additional rehabilitation, with review and progression at the weekly contact session. To ensure standardisation of the study rehabilitation protocol and interventions, training sessions took place with the trial physiotherapists at arranged visits.

\section{Physiotherapy review plus home exercise based regimen}

The home exercise based group comprised a minimum standard-of-care intervention that reflects the provision of postoperative physiotherapy across the UK. After the initial physiotherapist review, participants were instructed to adhere to a self-directed home exercise based protocol, which focused on unloaded bending of the knee to promote range of motion and using the weight of the limb to strengthen the quadriceps muscle (with a stationary knee). Eighteen self-directed sessions were performed over the six weeks (three times weekly).

\section{Assessments}

Study baseline assessment followed the clinical review six weeks post-surgery. Baseline data were collected before randomisation. We also collected outcome data at this point and then at 14, 26, and 52 weeks postsurgery by postal questionnaire. Reminder questionnaires were sent to non-responders after two weeks and followed-up by telephone if necessary. The physiotherapy teams collected additional physical performance data at weeks 8 and 14, before and after the six weeks of treatment intervention.

\section{Outcomes}

\section{Primary outcome}

We chose the patient reported Oxford knee score as the primary outcome because it specifically measures the outcomes of knee arthroplasty ${ }^{2021}$ and is routinely used in the UK. Scores range from 0 (worst) to 48 (best), with 4 points indicating a minimum clinically important difference. ${ }^{22}$

\section{Secondary outcomes}

Global knee pain severity was assessed using an 11 point (0-10) visual analogue scale, where 0 represents no pain and 10 the worst possible pain, with 1.1 points representing the minimum important clinical difference. ${ }^{23}$ The validity and sensitivity of the visual analogue scale has been well documented. ${ }^{24}$ We performed separate assessments of worst pain and of perceived mean daily pain, as recommended in clinical trials of osteoarthritis. ${ }^{25}$

Participants were asked to rate their satisfaction with the operated knee on a 4 point Likert scale (very satisfied, satisfied, unsure, or dissatisfied). Further single item questions inquired as to "how well the surgery relieved pain in the affected joint," "how well the surgery increased ability to perform regular activities," and "how well the surgery increased the ability to perform heavy work or sport activities" on the same 4 point Likert scale.

The timed-get-up-and-go test was performed before and after the intervention. This is a simple test used to assess an individual's mobility, requiring both static and dynamic balance. It is the time taken to rise from a chair, walk three metres, turn around, walk back to the chair, and sit down. The performance of this test has been found to decrease substantially with mobility impairments. $^{26} 27$

\section{Statistical analysis}

The primary endpoint was the 52 week Oxford knee score. During the development of the trial, the accepted minimum clinically important difference of this score was 3 points. ${ }^{21}$ Based on an $\alpha$ of 0.05 and an assumed standard deviation of 9.2, we determined that 300 patients would be required to detect a minimum clinically important difference of 3 points in Oxford knee score at 52 weeks with an $80 \%$ power and 400 patients for a $90 \%$ power. $^{16}$

We performed an intention-to-treat analysis, incorporating all randomised participants. The primary outcome was evaluated using analysis of covariance, with baseline Oxford knee score and study centre as covariates in a fixed effects model. For this we did not impute missing data. The initial analysis was confirmed using multiple imputation to explore the impact of missing data for the 52 week score. Post hoc analyses of the primary outcome included a longitudinal analysis of covariance including all time point data (baseline and 14, 26, and 52 weeks) to evaluate trajectory of recovery. We used the same analysis of covariance methods to evaluate secondary outcomes at the various postoperative time points.

We evaluated the analysis of covariance model of the timed-get-up-and go test with data recorded before and after the treatment intervention. Patient satisfaction was evaluated using ordinal regression, with baseline (sixth postoperative week) Oxford knee score, study centre, and the allocated intervention included as covariates.

Sensitivity analyses were performed on the per protocol sample using the same process. We defined adherence to the allocated intervention as no additional physiotherapy in the home exercise based group and as attendance of at least four of the six 
hospital based sessions in the therapist led outpatient intervention group.

The statistician conducting the analyses was blinded to group allocation. SAS (v9.2) was used for statistical analyses (SAS Institute), with two sided tests at a level of 0.05 considered to be significant.

\section{Protocol deviations}

The trial was originally powered to detect a 3 point difference in the Oxford knee score between intervention arms; the accepted minimum clinically important difference. Subsequent research (during trial recruitment) suggested that a 4 point difference would be required to show an observable or meaningful difference in patient outcome. ${ }^{22}$ As such we elected to interpret our trial with the newly defined 4 point difference. The independent trial steering committee approved this change. At the time of this change we had recruited 334 patients, and as the 4 point difference allowed in excess of $90 \%$ power to detect a between group difference, we closed recruitment. The health economic analysis for this trial will be reported separately.

\section{Patient and public involvement}

The study was developed in response to a survey of patients, which highlighted ambiguity and concern as to the correct amount of postoperative physiotherapy that should be undertaken after knee arthroplasty. The trial was developed at a workshop hosted by Arthritis Research UK (now Versus Arthritis) osteoarthritis clinical study group, which included surgical, physiotherapeutic, and patient representatives. The study steering group benefitted from a patient representative.

\section{Results}

Overall, 4264 patients were screened for eligibility of whom 334 were randomised. Most of the screened patients ( $n=2968,69.6 \%$ ) were not eligible for the study, enabling the rehabilitation intervention to be targeted to those defined as at risk of poor outcomes. Figure 1 outlines the reasons for non-recruitment. In addition to the 2968 ineligible patients, 572 declined to participate in the study. This number includes those who did not have equipoise to be randomised (ie, those who preferred a defined local rehabilitation), were unable to attend outpatient rehabilitation, and had no interest in taking part. In total, 390 patients were coded as other, which incorporated those taking part in another physiotherapy trial and problems with site logistics such as unavailability of research staff to recruit. The three patients with data missing on why they did not enrol are coded as other in the study flowchart.

Eight of the 334 randomised participants were lost to follow-up. Six participants withdrew from followup $(n=2(1.6 \%)$ therapist led group, $n=4(2.3 \%)$ home exercise based group). A further two participants (one $(0.6 \%)$ in each trial arm) were withdrawn from the study by their clinician.
Overall mean age of the randomised cohort was 67.5 (SD 9.46) years, 61.4\% $(n=205)$ were women, and overall mean body mass index was 31.34 (SD 5.67). The supplementary file lists the personal characteristics of screened and recruited patients. Table 1 summarises the baseline characteristics of the participants by study arms. Preoperative data for the primary outcome were available for a subset of centres; these data confirmed no difference in the average preoperative presentation between groups (see supplementary file).

Of the 334 randomised participants, 163 were allocated to therapist led outpatient rehabilitation and 171 to home exercise based rehabilitation. Compliance with treatment was $85.3 \%$ in the therapist led group and $97.7 \%$ in the home exercise group. The primary outcome was recorded for 148 (91\%) participants in the therapist led group and $156(91 \%)$ in the home exercise based group.

\section{Primary outcome}

In the intention-to-treat analysis, the adjusted mean between group difference in Oxford knee score at one year was 1.91 (95\% confidence interval -0.18 to 3.99) points favouring the therapist led group $(\mathrm{P}=0.07)$. The result was similar in the per protocol analysis (2.02, -0.15 to 4.18 ) points, $\mathrm{P}=0.07$ ).

\section{Secondary outcomes}

Both intervention arms showed an improvement in Oxford knee score (between baseline and 52 weeks) in excess of the 4 point minimum clinically important difference. When all time point data were analysed, the between group difference was 2.25 points $(95 \%$ confidence interval 0.61 to $3.90, \mathrm{P}=0.01$ ).

Postoperative differences in the timed-get-upand-go test were not significant when accounting for baseline scores (table 2 and supplementary file). Overall satisfaction did not differ between the groups (odds ratio 1.07, 95\% confidence interval 0.71 to 1.62); however, enhanced satisfaction with pain relief (1.66, 1.10 to 2.52$)$, ability to perform daily functional tasks $(1.66,1.09$ to 2.51$)$, and ability to perform heavy functional tasks $(1.57,1.02$ to 2.42$)$ was reported in the therapist led group (table 3 ).

\section{Planned secondary outcomes not reported in trial registration documents}

Small, non-significant reductions in worst and average pain scores were observed favouring the therapist led group. These reductions were substantially below the minimum clinically important difference of 1.1 points for this metric (table 2).

\section{Discussion}

In this study, no statistical or clinically meaningful differences were found for patient reported pain or functional outcomes in those at risk of poor outcomes after total knee arthroplasty between outpatient physiotherapy and a single physiotherapy review and home exercise based regimen. Overall satisfaction with the operated knee at one year did not differ between the 


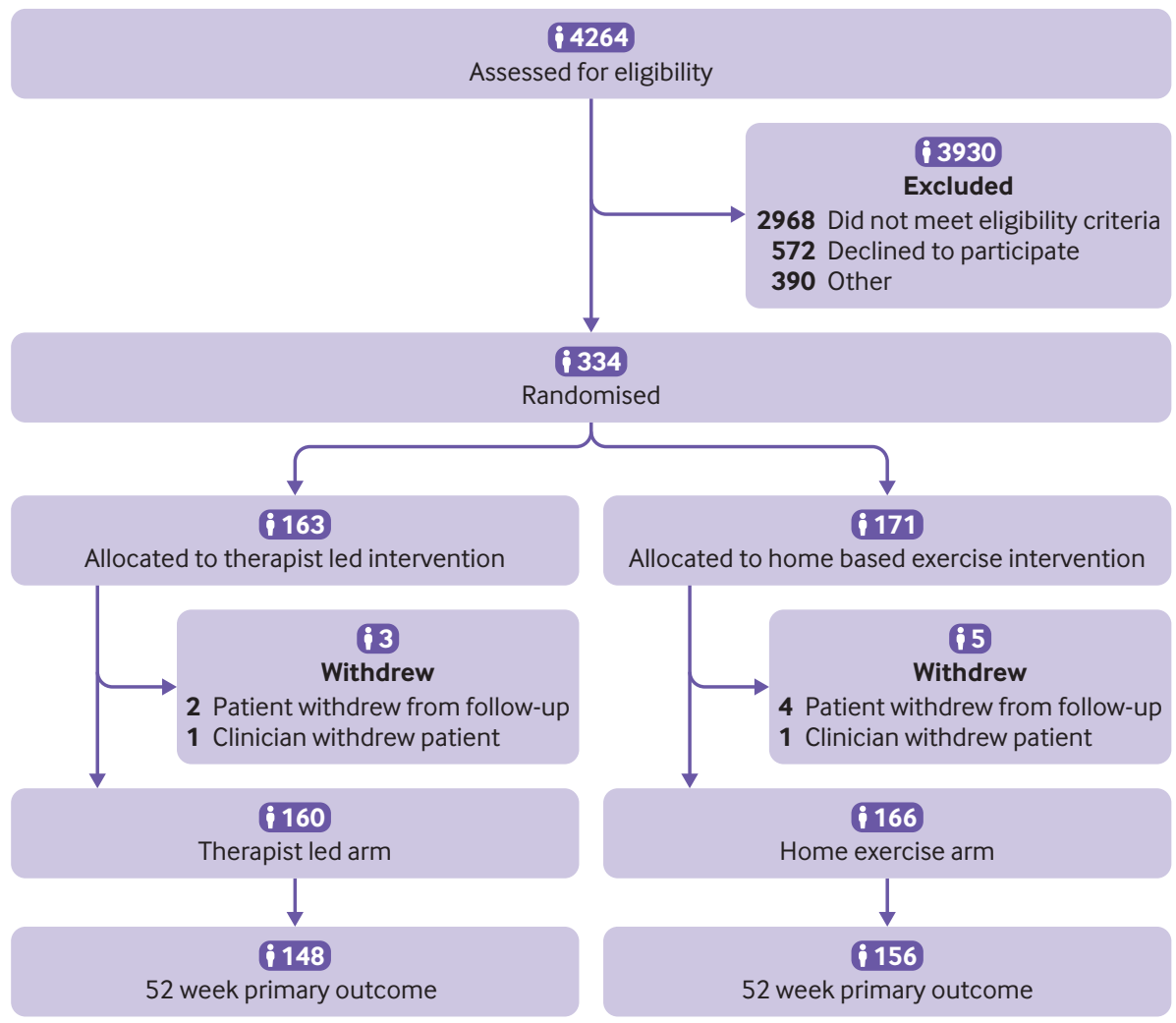

Fig 1 | Flow of participants through the study

groups; however, those who received the therapist led intervention reported enhanced satisfaction with pain relief and the ability to undertake physical activities.

\section{Strengths and limitations of this study}

Specific strengths of this study include the comparatively large sample size and low losses to-follow-up, which along with good treatment adherence ensured high study power to detect a between group difference.
One limitation is that we did not include a no treatment group for comparison. Although there is debate about the effectiveness of physiotherapy after total knee arthroplasty, and ambiguity about the best delivery method, it remains an accepted component of the treatment pathway for knee arthroplasty in the UK. As such it was considered unethical to include a control group of high risk participants who would not receive physiotherapy. As is the case with most physiotherapy

\begin{tabular}{|c|c|c|c|}
\hline Characteristics & Overall $(n=334)$ & Therapist led $(n=163)$ & Home exercise $(n=171)$ \\
\hline Mean (SD) age (years) & $67.5(9.46)$ & $66.8(9.46)$ & $68.2(9.44)$ \\
\hline Mean (SD) body mass index & $31.34(5.76)$ & $31.19(5.30)$ & $31.50(6.18)$ \\
\hline \multicolumn{4}{|l|}{ Comorbidity*: } \\
\hline Hypertension & $154(46.1)$ & $73(44.8)$ & $81(47.4)$ \\
\hline Lung disease & $45(13.5)$ & $20(12.3)$ & $25(14.6)$ \\
\hline Stroke & $11(3.3)$ & $6(3.7)$ & $5(2.9)$ \\
\hline Kidney disease & $2(0.6)$ & $1(0.6)$ & $1(0.6)$ \\
\hline Liver disease & $2(0.6)$ & $0(0)$ & $2(1.2)$ \\
\hline Other pain & $147(44)$ & $74(45.4)$ & $73(42.7)$ \\
\hline Mean (SD) Oxford knee score & $20.24(4.78)$ & $20.40(4.82)$ & $20.08(4.74)$ \\
\hline Mean (SD) timed-get-up-and-go & $21.66(63.13)$ & $18.85(44.8)$ & $24.33(80.6)$ \\
\hline Mean (SD) worst pain & $6.15(1.84)$ & $6.06(1.88)$ & $6.23(1.81)$ \\
\hline Mean (SD) average pain & $4.82(1.77)$ & $4.71(1.76)$ & $4.93(1.77)$ \\
\hline
\end{tabular}




\begin{tabular}{|c|c|c|c|c|c|c|}
\hline \multirow[b]{2}{*}{ Outcomes } & \multicolumn{2}{|c|}{ No of participants } & \multicolumn{2}{|c|}{ Mean (SD) } & \multirow{2}{*}{$\begin{array}{l}\text { Mean }(95 \% \mathrm{Cl}) \text { between } \\
\text { group difference* }\end{array}$} & \multirow[b]{2}{*}{$P$ for difference } \\
\hline & Therapist led & Home exercise & Therapist led & Home exercise & & \\
\hline \multicolumn{7}{|l|}{ Primary outcome } \\
\hline \multicolumn{7}{|c|}{ Oxford knee score at 52 weeks: } \\
\hline Intention to treat & 148 & 156 & $33.55(10.06)$ & $31.57(9.68)$ & $1.91(-0.18$ to 3.99$)$ & 0.07 \\
\hline Per protocol & 148 & 151 & $33.76(9.90)$ & $31.56(9.75)$ & $2.02(-0.15$ to 4.18$)$ & 0.07 \\
\hline \multicolumn{7}{|l|}{ Secondary outcomes } \\
\hline \multicolumn{7}{|l|}{ Oxford knee score: } \\
\hline 14 weeks & 154 & 151 & $31.80(7.64)$ & $30.20(8.13)$ & $1.60(0.05$ to 3.16$)$ & 0.04 \\
\hline 26 weeks & 150 & 151 & $32.12(8.81)$ & $30.34(8.75)$ & $1.70(-0.11$ to 3.51$)$ & 0.07 \\
\hline $\begin{array}{l}\text { Timed-get-up-and-go } \\
\text { (seconds) at } 14 \text { weeks }\end{array}$ & 143 & 143 & $14.65(38.0)$ & $22.5(77.2)$ & $4.64(-14.25$ to 4.96$)$ & 0.34 \\
\hline \multicolumn{7}{|c|}{ Outcomes not listed in trial registration documents } \\
\hline \multicolumn{7}{|c|}{ Worst pain: } \\
\hline 14 weeks & 154 & 150 & $3.97(2.46)$ & $4.44(2.41)$ & $0.46(-0.98$ to 0.07$)$ & 0.09 \\
\hline 26 weeks & 148 & 148 & $3.80(2.61)$ & $4.22(2.55)$ & $0.32(-0.86$ to 0.22$)$ & 0.24 \\
\hline 52 weeks & 147 & 156 & $3.36(2.92)$ & $3.64(2.80)$ & $0.22(-0.71$ to 0.41$)$ & 0.50 \\
\hline \multicolumn{7}{|l|}{ Average pain: } \\
\hline 14 weeks & 154 & 150 & $2.87(2.09)$ & $3.21(2.06)$ & $0.29(-0.71$ to 0.14$)$ & 0.19 \\
\hline 26 weeks & 147 & 147 & $3.02(2.21)$ & $3.45(2.25)$ & $0.26(-0.72$ to 0.19$)$ & 0.25 \\
\hline 52 weeks & 147 & 154 & $2.72(2.52)$ & $3.09(2.51)$ & $0.25(-0.78$ to 0.28$)$ & 0.36 \\
\hline
\end{tabular}

trials, it was not feasible to blind patients or therapists to treatment allocation. To ensure generalisability and limit excess treatment costs, we compared physiotherapy interventions that are deliverable within the constraints of national health service resources. Standard commissioning in the NHS limits physiotherapy provision to about six sessions, therefore we developed our protocols to reflect this provision. A more intensive rehabilitation intervention might have had a beneficial effect; however, studies utilising more comprehensive and lengthy rehabilitation protocols have also found no benefit. ${ }^{10-15}$ Responder bias was possible as the outcomes were primarily patient reported, although such bias would probably favour the therapist led arm as physiotherapy contact was greater, and the generalisability to other healthcare settings outside the NHS is assumed but undefined.

\section{Implications for patients and policy}

Consensus as to the best way to deliver rehabilitation after total knee arthroplasty is lacking. Specific protocols are strongly entrenched at individual physiotherapy departments despite the wider efficacy of postoperative physiotherapy being poorly established. ${ }^{28}$ This uncertainty about effectiveness of physiotherapy makes it difficult for patients, commissioning organisations, and healthcare provi- ders to determine the best way to deliver physiotherapy after total knee arthroplasty and the correct level and mechanism of funding for such services.

It is increasingly evident that patient outcomes one year after total knee arthroplasty are not influenced by the location or type of postoperative rehabilitation when applied to all patients. ${ }^{10-15}$ We targeted physiotherapy interventions specifically at patients considered to be at most risk of poor outcomes. Predicting preoperatively which patients will do well or poorly after total knee arthroplasty has proved challenging despite the large numbers of patients who undergo this procedure annually. Personal factors relating to patient heath and symptoms are a useful general guide, although these preoperative indicators do not predict those who will benefit from physiotherapy postoperatively. ${ }^{9}$ We therefore recruited poorly performing patients on the basis of actual functional status at the time of early (six week) postoperative clinical review, targeting our interventions to those presenting with higher than normal levels of knee specific pain, finding it difficult to perform simple tasks of daily living, and most vulnerable to experiencing poor clinical outcomes.

Why some patients experience poorer recovery after knee arthroplasty is not well understood and might suggest that physiotherapy exerts a marginal influence on the underlying causes of poor post-

\begin{tabular}{|c|c|c|c|c|}
\hline \multirow[b]{2}{*}{ Satisfaction question } & \multicolumn{2}{|c|}{ Positive response } & \multirow{2}{*}{$\begin{array}{l}\text { Odds ratio }(95 \% \mathrm{Cl}) \\
\text { for difference* }\end{array}$} & \multirow[b]{2}{*}{ P for difference } \\
\hline & Therapist led & Home exercises & & \\
\hline How satisfied are you with your operated knee? & $95(58.3)$ & $90(52.6)$ & $1.07(0.71$ to 1.62$)$ & 0.75 \\
\hline \multicolumn{5}{|l|}{ How well did the surgery: } \\
\hline relieve the pain in your affected joint? & $108(66.3)$ & $94(55.0)$ & $1.66(1.10$ to 2.52$)$ & 0.02 \\
\hline increase your ability to perform regular activities? & $89(54.6)$ & 75 (43.9) & 1.66 (1.09 to 2.51$)$ & 0.02 \\
\hline allow you to perform heavy work or sports activities? & $46(28.3)$ & $43(25.1)$ & 1.57 (1.02 to 2.42$)$ & 0.04 \\
\hline
\end{tabular}


surgery outcomes-for example, functional limitations that might relate to implant positioning, and intraoperative factors that might not be modifiable with postoperative physiotherapy (irrespective of content). Other patient factors such as comorbid conditions, local muscle deficits, and attitudes towards health or catastrophising behaviours might also limit responsiveness to postoperative rehabilitation.

Although we selectively recruited only those considered at risk of poor outcomes, the mean (trial cohort) Oxford Knee Score at one year was 4-6-points below the wider UKaverage of 36 points ${ }^{29}$ for all patients after knee arthroplasty, suggesting that even those who show poorer recovery in the early postoperative phase can achieve reasonable outcomes by one year with a rehabilitation intervention and that the method of delivery and content of the physiotherapy received might be comparatively unimportant. It could be that simply making patients aware that they are at risk of poor outcomes and providing hands-off rehabilitation through physiotherapist review and a home exercise based regimen are enough to optimise outcomes; in this study we found no benefit of providing an alternative more comprehensive physiotherapist facilitated outpatient rehabilitation.

\section{Conclusions}

In this randomised trial targeting physiotherapy to patients at risk of poor outcomes after total knee arthroplasty, we observed no meaningful differences in patient outcome or overall satisfaction with outcome at one year as a result of patients undertaking outpatient physiotherapy compared with a single physiotherapy review and home exercise based regimen. Although targeting rehabilitation interventions to at risk patients is a feasible delivery method, the content of the rehabilitation seems to have minimal influence on patient outcomes.

\section{AUTHOR AFFILIATIONS}

${ }^{1}$ Department of Orthopaedics and Trauma, University of Edinburgh, Edinburgh EH16 4SB, UK

${ }^{2}$ Nuffield Department of Orthopaedics, Rheumatology and Musculoskeletal Sciences, University of Oxford, Oxford, UK ${ }^{3}$ Aberdeen Centre for Arthritis and Musculoskeletal Health (Epidemiology) Group, University of Aberdeen, Aberdeen, UK ${ }^{4}$ Usher Institute of Population Health, University of Edinburgh, Edinburgh, UK

${ }^{5}$ Department of Orthopaedics, Royal Derby Hospital, Derby, UK ${ }^{6}$ Department of Orthopaedic Surgery, University of Aberdeen, Aberdeen, UK

We thank the study participants and the investigators, physiotherapists, and research teams for delivering the study at the participating centres; the Edinburgh Clinical Trials Unit for coordinating the project, in particular Chris Tuck, Elaine Kinsella, and Philip Rayson (trial management team), Valentina Assi (statistics), Ronnie Harkess (programming), and Lyndsey Milne (data management); and the support and advice at all study stages of the independent trial oversight and data monitoring committee: Alex MacGregor (University of East Anglia), Martin Lewis (Keele University), Michael Docherty (University of Nottingham), and Merfyn Williams (lay member).

TRIO investigators include: Caroline Austrie, the Royal Free Hospital, London; Kim Brown, Rehabilitation Centre, Queen Alexandria Hospital, Portsmouth; Matthew Carr, the Horder Centre, Crowborough; Ivan Brenkel, Victoria Hospital, Kirkcaldy; Tom Briggs,
Doncaster Royal Infirmary, Doncaster; Katherine Dillow, Daren’t Valley Hospital, Dartford; Jamila Kassam, Royal London Hospital, London; Ben Lankester, Yeovil District Hospital, Yeovil; Emma McLoughlin, Rehabilitation Centre, Queen Alexandria Hospital, Portsmouth; Helen Samuel (Nee Sankey), the Tunbridge Wells Hospital, Pembury; Jason Seaton, Weston General Hospital, Weston Super Mare; Kate Weatherly, Conquest Hospital, St. Leonards-on-Sea.

Contributors: DH and HS conceived the study. All authors designed the study. GM and AS conducted the analysis. DH drafted the manuscript. All authors interpreted the data, critically revised the manuscript, and approved the final draft. HS is the guarantor. The corresponding author attests that all listed authors meet authorship criteria and that no others meeting the criteria have been omitted.

Funding: This study was funded by Arthritis Research UK (now Versus Arthritis), ref 20100, and sponsored by ACCORD (the University of Edinburgh and NHS Lothian). The funder had no role in study design, data collection, data analysis, data interpretation, writing of the article, or the decision to submit the paper for publication.

Competing interests: All authors have completed the ICMJE uniform disclosure form at www.icmje.org/coi_disclosure.pdf and declare: funding from Arthritis Research UK; no financial relationships with any organisations that might have an interest in the submitted work in the previous three years; no other relationships or activities that could appear to have influenced the submitted work.

Ethical approval: The trial protocol was approved by the South East Scotland Research Ethics Committee 01 (13/SS/0051) and local research and development departments at participating sites.

Data sharing: Anonymised patient level data can be made available on reasonable request and after approval by a review panel. Requests should be made to ECTUdatashare@ed.ac.uk.

The lead author affirms that the manuscript is an honest, accurate, and transparent account of the study being reported; that no important aspects of the study have been omitted; and that any discrepancies from the study as originally planned (and, if relevant, registered) have been explained.

Dissemination to participants and related patient and public communities: We intend to disseminate the results of this study to the participants through Versus Arthritis and the local hospital physiotherapy departments.

Provenance and peer review: Not commissioned; externally peer reviewed.

This is an Open Access article distributed in accordance with the terms of the Creative Commons Attribution (CC BY 4.0) license, which permits others to distribute, remix, adapt and build upon this work, for commercial use, provided the original work is properly cited. See: http://creativecommons.org/licenses/by/4.0/

1 National Joint Registry. 14th annual report 2017. National Joint Registry for England, Wales, Northern Ireland and the Isle of Man: surgical data to 31 December 2016. 2017. www.hqip.org.uk/ resource/national-joint-registry-14th-annual-report-2017/\#. X2Xj9WhKiUk (accessed lanuary 17th, 2019).

2 Price AJ, Alvand A, Troelsen A, et al. Knee replacement. Lancet 2018;392:1672-82. doi:10.1016/S0140-6736(18)32344-4

3 Kurtz S, Ong K, Lau E, Mowat F, Halpern M. Projections of primary and revision hip and knee arthroplasty in the United States from 2005 to 2030. J Bone Joint Surg Am 2007;89:780-5. doi:10.2106/00004623-200704000-00012

4 Inacio MCS, Paxton EW, Graves SE, Namba RS, Nemes S. Projected increase in total knee arthroplasty in the United States - an alternative projection model. Osteoarthritis Cartilage 2017;25:1797803. doi:10.1016/j.joca.2017.07.022

5 Culliford D, Maskell J, Judge A, Cooper C, Prieto-Alhambra D, Arden NK, COASt Study Group. Future projections of total hip and knee arthroplasty in the UK: results from the UK Clinical Practice Research Datalink. Osteoarthritis Cartilage 2015;23:594-600. doi:10.1016/j. joca.2014.12.022

6 Hamilton DF, Lane JV, Gaston P, et al. What determines patient satisfaction with surgery? A prospective cohort study of 4709 patients following total joint replacement. BMJ Open 2013;3:e002525. doi:10.1136/bmjopen-2012-002525

7 Naylor JM, Hart A, Harris IA, Lewin AM. Variation in rehabilitation setting after uncomplicated total knee or hip arthroplasty: a call for evidence-based guidelines. BMC Musculoskelet Disord 2019;20:214. doi:10.1186/s12891-019-2570-8

8 Buhagiar A, Naylor JM, Harris I, Xuan W, Kohler F, Wright R, Fortunato R. Effect of Inpatient Rehabilitation vs a Monitored Home-Based Program on Mobility in Patients with Total Knee Arthroplasty The HIHO Randomized Clinical Trial. JAMA 2017;317:1037-46. 
9 Hamilton DF, Loth FC, MacDonald DJ, MacFarlane GJ, Beard DJ, Simpson AHR, Patton JT, Howie CR. Exploring variation in patient access of post-discharge physiotherapy following total hip and knee arthroplasty under a choice-based system in the UK: an observational cohort study. BMJ Open 2019;9:e021614.

10 Smith TO, Dainty JR, Clark EM, Whitehouse MR, Price AJ, MacGregor AJ. Demographic and geographical variability in physiotherapy provision following hip and knee replacement. An analysis from the National Joint Registry for England, Wales, Northern Ireland and the Isle of Man. Physiotherapy 2020;106:1-11. doi:10.1016/j. physio.2019.11.003

11 Khan F, Ng L, Gonzalez S, Hale T, Turner-Stokes L. Multidisciplinary rehabilitation programmes following joint replacement at the hip and knee in chronic arthropathy. Cochrane Database Syst Rev 2008;2:CD004957. doi:10.1002/14651858.CD004957.pub3

12 Artz N, Elvers KT, Lowe CM, Sackley C, Jepson P, Beswick AD. Effectiveness of physiotherapy exercise following total knee replacement: systematic review and meta-analysis. BMC Musculoskelet Disord 2015;16:15. doi:10.1186/s12891-0150469-6

13 Minns Lowe C), Barker KL, Dewey M, Sackley CM. Effectiveness of physiotherapy exercise after knee arthroplasty for osteoarthritis: systematic review and meta-analysis of randomised controlled trials. BMJ 2007;335:812. doi:10.1136/bmj.39311.460093.BE

14 Buhagiar MA, Naylor JM, Harris IA, Xuan W, Adie S, Lewin A. Assessment of Outcomes of Inpatient or Clinic-Based vs Home-Based Rehabilitation After Total Knee Arthroplasty: A Systematic Review and Meta-analysis. JAMA Netw Open 2019;2:e192810. doi:10.1001/ jamanetworkopen.2019.2810

15 Florez-García M, García-Pérez F, Curbelo R, et al. Efficacy and safety of home-based exercises versus individualized supervised outpatient physical therapy programs after total knee arthroplasty: a systematic review and meta-analysis. Knee Surg Sports Traumatol Arthrosc 2017;25:3340-53. doi:10.1007/s00167-016-4231-x

16 Simpson AHRW, Hamilton DF, Beard DJ, et al. Targeted rehabilitation to improve outcome after total knee replacement (TRIO): study protocol for a randomised controlled trial. Trials 2014;15:44. doi:10.1186/1745-6215-15-44

17 Kalairajah Y, Azurza K, Hulme C, Molloy S, Drabu KJ. Health outcome measures in the evaluation of total hip arthroplasties--a comparison between the Harris hip score and the Oxford hip score. Arthroplasty 2005;20:1037-41. doi:10.1016/i.arth.2005.04.017

18 Rothwell AG, Hooper GJ, Hobbs A, Frampton CM. An analysis of the Oxford hip and knee scores and their relationship to early joint revision in the New Zealand Joint Registry. J Bone Joint Surg Br 2010:92:413-8 doi:10.1302/0301-620X.92B3.22913

19 Piva SR, Gil AB, Almeida GJ, DiGioia AM3rd, Levison TJ, Fitzgerald GK. A balance exercise program appears to improve function fo patients with total knee arthroplasty: a randomized clinical trial. Phys Ther 2010;90:880-94. doi:10.2522/ptj.20090150

20 Dawson J, Fitzpatrick R, Murray D, Carr A. Questionnaire on the perceptions of patients about total knee replacement. J Bone Joint Surg Br 1998;80:63-9. doi:10.1302/0301-620X.80B1.0800063

21 Murray DW, Fitzpatrick R, Rogers K, et al. The use of the Oxford hip and knee scores. J Bone Joint Surg Br 2007;89:1010-4. doi:10.1302/0301-620X.89B8.19424

22 Beard DJ, Harris K, Dawson J, et al. Meaningful changes for the Oxford hip and knee scores after joint replacement surgery. J Clin Epidemiol 2015;68:73-9. doi:10.1016/j.jclinepi.2014.08.009

23 Hawker GA, Mian S, Kendzerska T, French M. Measures of adult pain: Visual Analog Scale for Pain (VAS Pain), Numeric Rating Scale for Pain (NRS Pain), McGill Pain Questionnaire (MPQ), Short-Form McGill Pain Questionnaire (SF-MPQ), Chronic Pain Grade Scale (CPGS), Short Form-36 Bodily Pain Scale (SF-36 BPS), and Measure of Intermittent and Constant Osteoarthritis Pain (ICOAP). Arthritis Care Res (Hoboken) 2011;63(Suppl 11):S240-52. doi:10.1002/ acr.20543

24 Von Korff M, Jensen MP, Karoly P. Assessing global pain severity by self-report in clinical and health services research. Spine (Phila Pa 1976) 2000;25:3140-51. doi:10.1097/00007632-20001215000009

25 Perrot S, Rozenberg S, Moyse D, Legout V, Marty M, cercle d'étude de douleur en rhumatologie (CEDR). Comparison of daily, weekly or monthly pain assessments in hip and knee osteoarthritis. A 29-day prospective study. Joint Bone Spine 2011;78:510-5. doi:10.1016/j. jbspin.2010.11.009

26 Bischoff HA, Stähelin HB, Monsch AU, et al. Identifying a cut-off point for normal mobility: a comparison of the timed 'up and go' test in community-dwelling and institutionalised elderly women. Age Ageing 2003;32:315-20. doi:10.1093/ageing/32.3.315

27 Ng SS, Hui-Chan CW. The timed up \& go test: its reliability and association with lower-limb impairments and locomotor capacities in people with chronic stroke. Arch Phys Med Rehabil 2005;86:1641-7. doi:10.1016/i.apmr.2005.01.011

28 Beard D, Hamilton D, Davies L, et al. Evidence-based evaluation of practice and innovation in Physical Therapy using the IDEAL-Physio framework. Phys Ther 2018;98:108-21. doi:10.1093/pti/pzx103

29 NHS Digital. Finalised Patient Reported Outcome Measures (PROMs) in England for Hip \& Knee Replacements, April 2018 - March 2019. 2020. https://digital.nhs.uk/data-and-information/publications/ statistical/patient-reported-outcome-measures-proms/finalised-hip knee-replacements-april-2018-march-2019 (last accessed 27 Feb 2020)

Supplementary information: additional tables 